13

41-55

\author{
Valencia \\ (España)
}

\title{
Aplicación de la topología molecular a la predicción de la actividad antimalárica de análogos de la 4-anilinoquinolina
}

\author{
Application of molecular topology to the prediction of \\ antimalarial activity of 4-anilinoquinoline analogues
}

Fecha de recepción y aceptación: 17 de febrero de 2021 y 13 de abril de 2021

DOI: $10.46583 /$ nereis_2021.13.811

\author{
A. E. Peñarrubia ${ }^{1}$ R. Peña1, J. Mayorga ${ }^{1}$, A. Urbanska ${ }^{1}$, J. Gálvez ${ }^{2}$ y R. García-Doménech ${ }^{2 *}$ \\ ${ }^{1}$ Departamento de Farmacia y Tecnología Farmacéutica y Parasitología. Facultad de Farmacia. Universitat de València. \\ ${ }^{2}$ Departamento de Química Física. Facultad de Farmacia. Universitat de València. \\ * Correspondencia: Universitat de València. Facultad de Farmacia. Departamento de Química Física. Avenida V. A. Estellés, s/n. \\ 46100 (Burjassot), Valencia. España.E-mail: ramon.garcia@uv.es
}

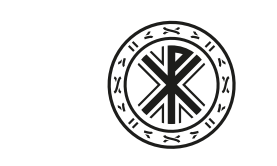

Universidad

Católica de Valencia San Vicente Mártir

\section{RESUMEN}

La malaria es una enfermedad parasitaria causada por protozoos del género Plasmodium y transmitida por vectores del género Anopheles. En 2019 esta enfermedad se cobró la vida de más de 400.000 personas, de las cuales un 94 \% se concentraban en la región de África. Uno de los principales problemas en el control de la malaria es la aparición de resistencias frente a los diferentes fármacos que existen, es por ello que es necesario el desarrollo de alternativas antimaláricas eficaces. En este estudio se ha aplicado la topología molecular a una serie de compuestos análogos de la 4-anilinoquinolina con actividad inhibitoria de la proliferación de 3 cepas de Plasmodium falciparum, una sensible a la cloroquina (D6) y dos resistentes (W2 y C235); para así desarrollar un modelo de relación cuantitativa estructura-actividad QSAR que permita predecir la actividad de los compuestos frente a cada una de ellas. Utilizando el análisis lineal discriminante se seleccionaron tres funciones que clasificaron correctamente el $87 \%$ de los compuestos analizados en la cepa D6, en la W2 y en la C235, respectivamente. Para la validación del modelo se empleó un test interno del tipo leave some out para cada una de las funciones. Por último, se aplicó el modelo en la búsqueda de nuevos compuestos antimaláricos potencialmente activos frente a las tres cepas.

PALABRAS CLAVE: Topología molecular, análisis QSAR, Malaria, Plasmodium falciparum.

\section{ABSTRACT}

Malaria is a parasitic disease caused by the Plasmodium protozoa and transmitted by vector of the genus Anopheles. In 2019, this disease claimed the lives of more than 400.000 people, of which $94 \%$ are concentrated in the African region. One of the main problems with malaria control is parasite resistance to the different drugs that exist, which is why it is necessary to develop effective antimalarial alternatives. In this study, molecular topology was applied to 4-anilinoquinoline analogs with proliferation inhibitory activity of 3 Plasmodium falciparum strains, one 
chloroquine sensitive (D6) and two chloroquine resistant (W2 and C235); in order to develop a quantitative structure-activity (QSAR) model to predict the activity of the compounds against each of them. Using linear discriminant analysis, three functions were selected that correctly classified $87 \%$ of the compounds analyzed in strain D6, W2 and C235, respectively. The leave some out test was carried out to validate this model. Finally, the model was applied to search for new antimalarial compounds potentially active against all three strains.

KEYWORDS: Molecular topology, QSAR Analysis, Malaria, Plasmodium falciparum.

\section{INTRODUCCIÓN}

La malaria, o paludismo, es una enfermedad parasitaria potencialmente mortal y endémica de los países tropicales. Está causada por parásitos del género Plasmodium, que se transmiten al ser humano mediante la picadura de las hembras de mosquitos del género Anopheles, los denominados vectores de la enfermedad. A pesar de que se puede prevenir y tratar, se estima que en 2019 hubo 229 millones de casos de paludismo en 87 países y las muertes estaban alrededor de las 409.000, siendo la región de África de la OMS la más afectada con un $94 \%$ de los casos y de los fallecimientos [1].

No obstante, cabe destacar que, a nivel mundial, la tasa de incidencia de la mortalidad por malaria (muertes por cada 100.000 habitantes en riesgo) se redujo de 25 en el año 2000 a 12 en 2015 y 10 en 2019, con una desaceleración en la tasa de disminución en los últimos años debido, entre otros factores, a la aparición de resistencias frente a los tratamientos e insecticidas empleados para el control de la enfermedad [2].

Desde su desarrollo en la década de 1930, la cloroquina ha sido el tratamiento de referencia, ya que resultó ser altamente efectiva, barata de producir y de baja toxicidad, pero su administración incontrolada hizo que surgiera la resistencia hacia este medicamento, la cual se extendió rápidamente por todo el mundo. Actualmente, las terapias combinadas basadas en artemisinina (TCA) son el tratamiento activo más rápido y eficaz disponible, especialmente para el paludismo no severo causado por P. falciparum [3]. Sin embargo, ya se han detectado también resistencias parciales a esta en la subregión del Gran Mekong [1].

Todo esto hace plantearse la necesidad de encontrar nuevos fármacos efectivos para el tratamiento de esta enfermedad. Una manera económica, rápida y sencilla de hacerlo es generando modelos basados en la relación cuantitativa estructura-actividad (QSAR, del inglés quantitative structure-activity relationship) de las moléculas y utilizando la topología molecular, una aproximación que describe matemáticamente las estructuras moleculares, a través de los índices topológicos (IT) y haciendo uso de objetos matemáticos como grafos y matrices [4].

La topología molecular se ha aplicado con éxito en el campo antimalárico [5-7] y en la lucha contra los vectores transmisores de enfermedades parasitarias $[8,9]$.

En un estudio reciente en el que se ha optimizado la actividad antimalárica de grupos de compuestos análogos de la 4-anilinoquinolina con efecto inhibidor de la proliferación del parásito, se realizaron estudios de actividad frente a distintas cepas de P. falciparum, una sensible a la cloroquina (cepa D6) y dos cepas resistentes (cepa W2 y cepa C235) [10]. 
De esta manera, usando las ecuaciones de correlación adecuadas entre las propiedades experimentales de los compuestos presentados en el trabajo y sus índices topológicos (IT) calculados, es posible no solo predecir las propiedades de las sustancias químicas conocidas, sino también diseñar otras nuevas sin necesidad de un conocimiento explícito de su mecanismo de acción, lo cual representa una gran ventaja frente a los métodos convencionales [4].

El objetivo de este trabajo es desarrollar y evaluar un modelo topológico-matemático de predicción QSAR, usando el análisis discriminante basado en la topología molecular para poder identificar nuevos compuestos activos contra las tres cepas de P. falciparum.

\section{MATERIALES Y MÉTODOS}

\section{Compuestos analizados}

En este estudio se utilizaron 54 compuestos análogos de la 4-anilinoquinolina con actividad inhibitoria de la proliferación de Plasmodium falciparum, cuya actividad experimental y concentración $\mathrm{EC}_{50}(\mu \mathrm{M})$ fueron determinadas por Mehta y colaboradores [10]. De este mismo estudio se obtuvieron los códigos SMILES de dichos compuestos para dibujar su estructura molecular utilizando el programa BIOVIA Draw (R) Versión MDL.Draw.Editor 17.1.0.900. [11] (tabla 1).

Tabla 1.Estructura química de los compuestos utilizados en este estudio

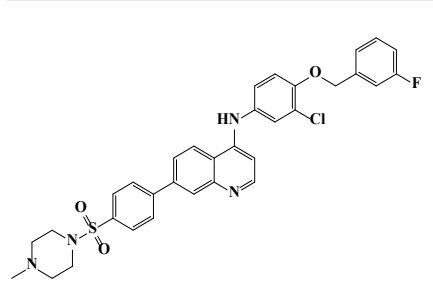

NEU-961

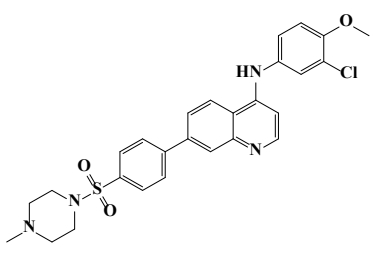

NEU-1029

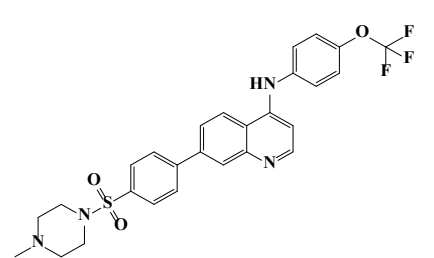

NEU-1927

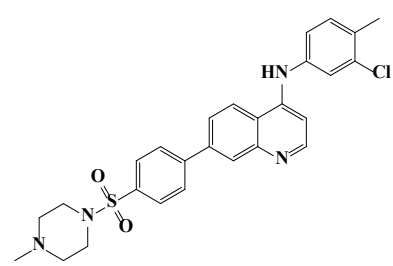

NEU-1031

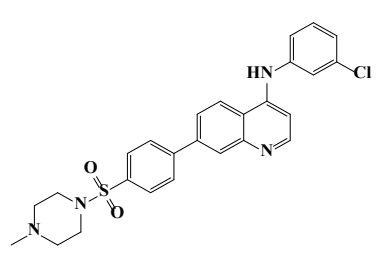

NEU-1030

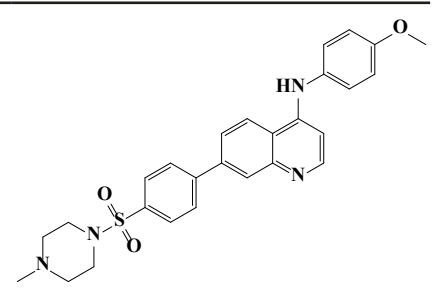

NEU-1926

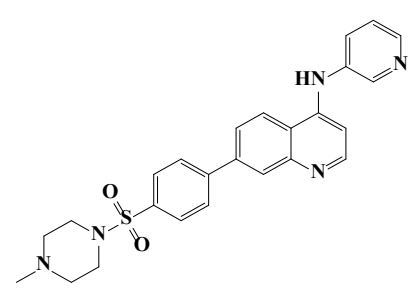

NEU-1019

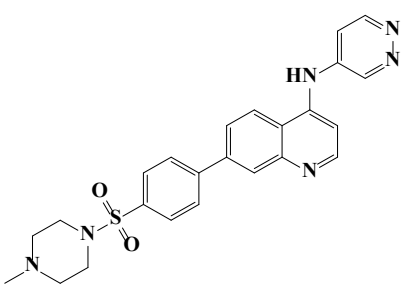

NEU-1020 


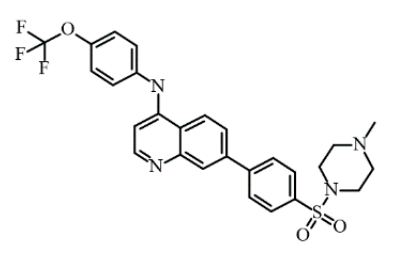

NEU-1027

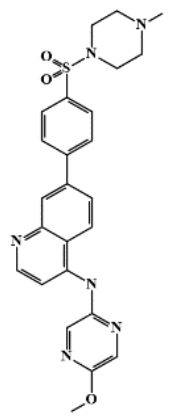

NEU-1022

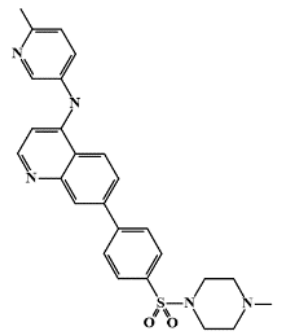

NEU-1929

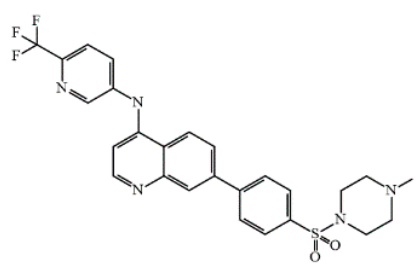

NEU-1930

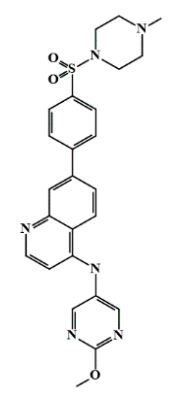

NEU-1021

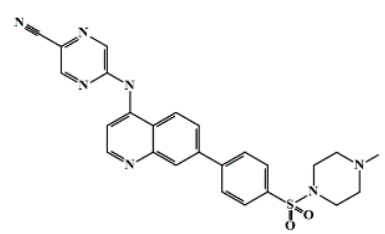

NEU-1024

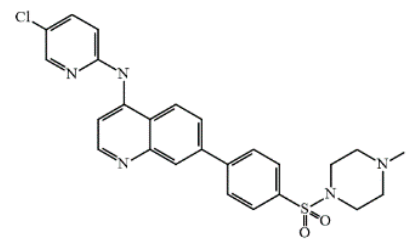

NEU-1026

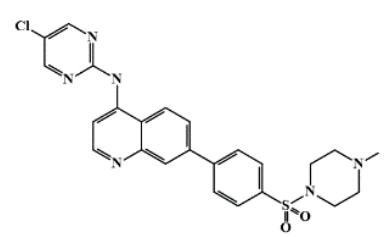

NEU-1025<smiles>CC1CCC(CC2CCCC3CC(C4CCC(C(=O)C5CCC(C)CC5)CC4)CCC23)CC1</smiles>

NEU-1028

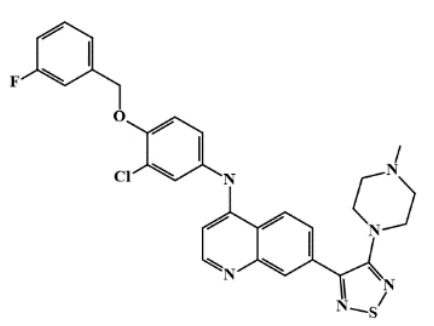

NEU-1899

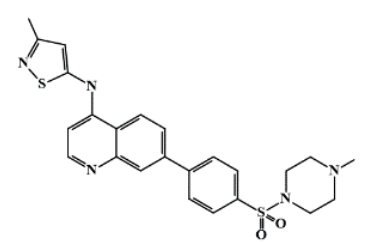

NEU-1023

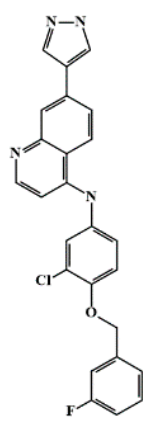

NEU-1900

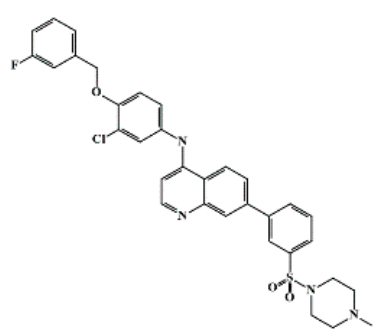

NEU-1931

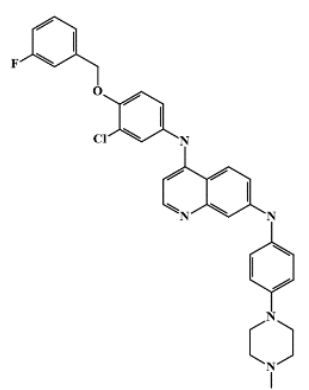

NEU-2076

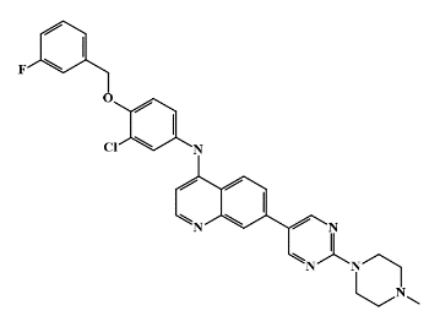

NEU-1060

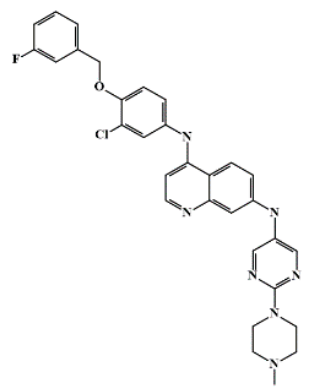

NEU-2152 
Aplicación de la topología molecular a la predicción de la actividad antimalárica...

45

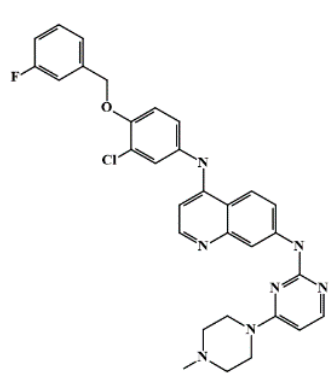

NEU-2086
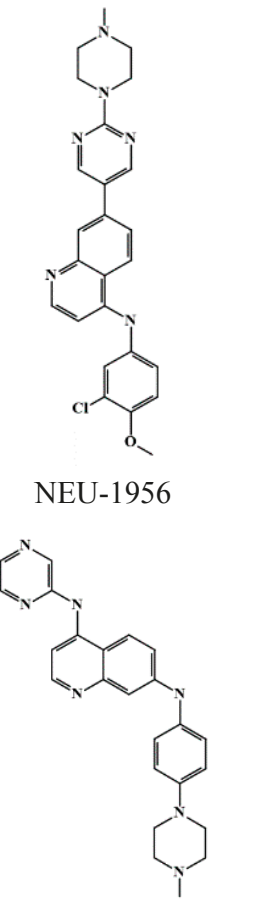

NEU-2096

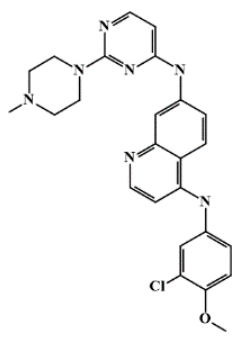

NEU-2093

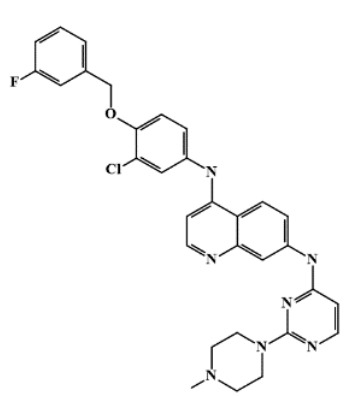

NEU-2077

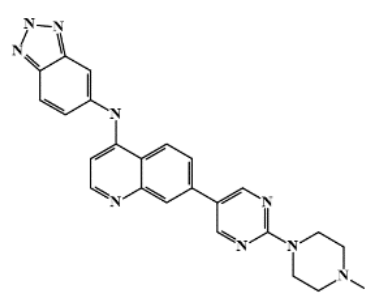

NEU-1957

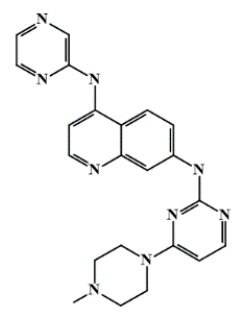

NEU-2091

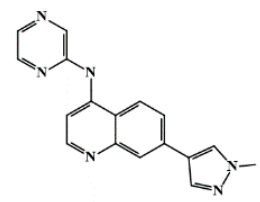

NEU-2129

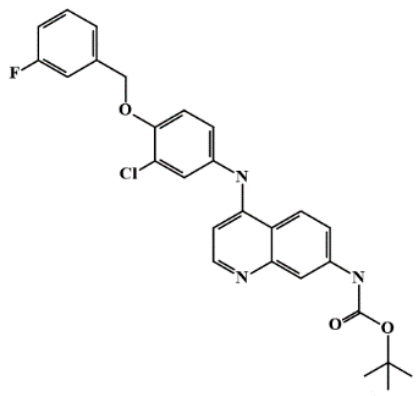

NEU-1898

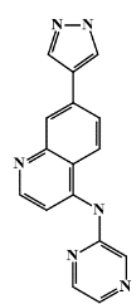

NEU-2088

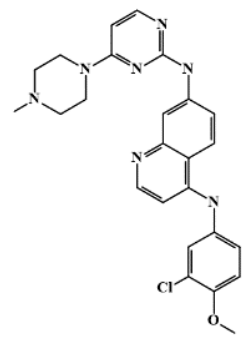

NEU-2090

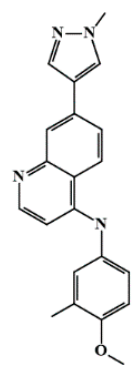

NEU-2130

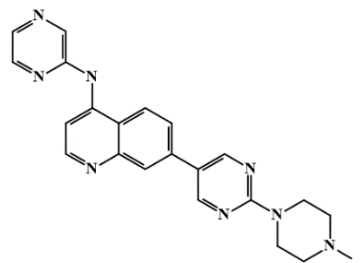

NEU-1953

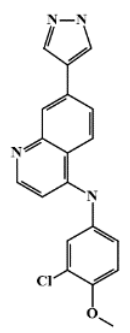

NEU-2087

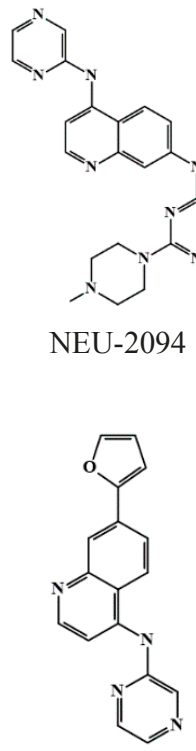

NEU-2131

NEREIS 13 [Marzo 2021], 41-55, ISSN: 1888-8550

來 
46

A. E. Peñarrubia, R. Peña, J. Mayorga, A. Urbanska, J. Gálvez y R. García-Doménech

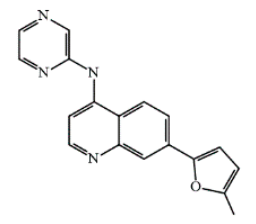

NEU-2133

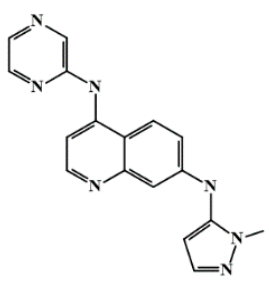

NEU-2144

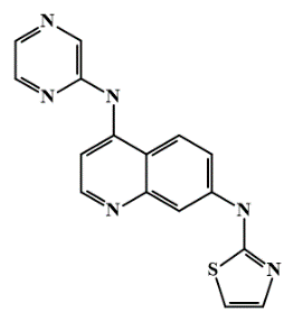

NEU-2141

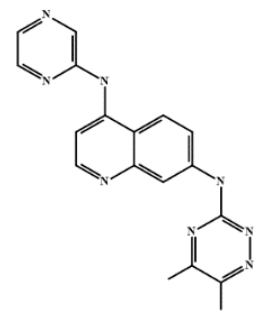

NEU-2151

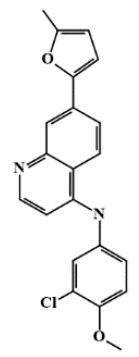

NEU-2134

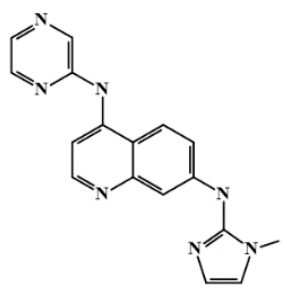

NEU-2147

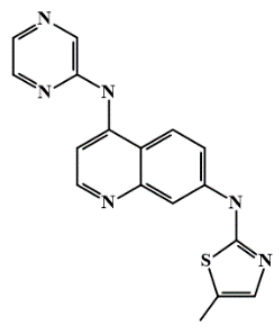

NEU-2149

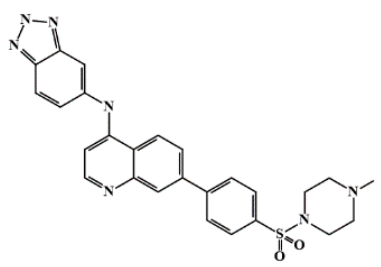

NEU-1018

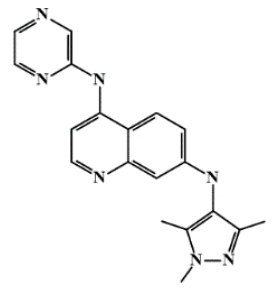

NEU-2142

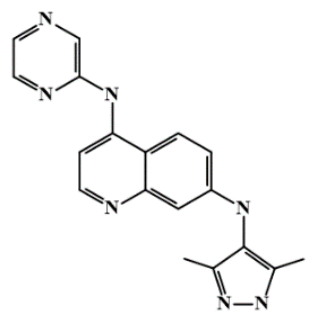

NEU-2146

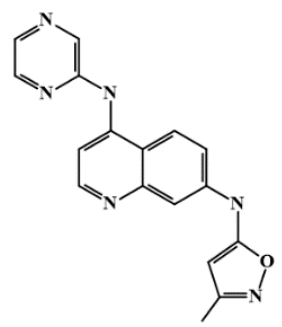

NEU-2150<smiles>CN1CC2CC(Nc3ccc4c(Nc5cnccn5)ccnc4c3)C=CN2C1</smiles>

NEU-2143<smiles>CC1CC(Nc2ccc3c(Nc4cnccn4)ccnc3c2)CN1</smiles>

NEU-2148<smiles>c1cnc(Nc2ccc3c(Nc4cnccn4)ccnc3c2)nc1</smiles>

NEU-2145

Descriptores moleculares

La estructura molecular de los 54 compuestos, se analizó mediante el programa DRAGON® versión 5.0. [12], que calculó alrededor de 500 descriptores topológicos ITS, de los cuales se seleccionaron los estadísticamente más significativos $(\mathrm{p}<0,05)$, que fueron usados para la obtención de las funciones discriminantes (FD), en las tres cepas de Plasmodium falciparum que se tuvieron en cuenta para este estudio.

NEREIS 13 [Marzo 2021], 41-55, ISSN: 1888-8550 
Algunos de los índices topológicos utilizados para caracterizar los compuestos fueron índices topológicos de carga de quinto y séptimo orden [13], índices tipo Balaban [14], valores propios de Burden, índices de los valores propios de la matriz de distancia, etc.

Los IT expresan de forma numérica la estructura molecular de cada compuesto, lo que facilita la identificación de sus propiedades fisicoquímicas y, a su vez, la búsqueda o el diseño de nuevos compuestos con propiedades similares [15].

\section{Algoritmos QSAR}

\section{Análisis lineal discriminante}

El análisis lineal discriminante (ALD) es empleado con el fin de encontrar una función lineal, capaz de discriminar entre compuestos activos e inactivos, partiendo de una concentración de referencia y de acuerdo con los valores obtenidos de los descriptores moleculares IT [16].

En este trabajo, se utilizó el software Statistica ${ }^{\circledR}$ versión 8.0 [17], para realizar un ALD con las tres cepas de P. falciparum, seleccionando FD altamente sensibles (con capacidad para detectar compuestos activos) y específicas (con capacidad para detectar compuestos inactivos), sobre la base de la actividad antimalárica de cada compuesto. Para las tres cepas, el fármaco de referencia fue la cloroquina, aunque una de ellas es sensible a este fármaco, las otras dos son resistentes. Por esta razón, tanto la concentración de referencia como el criterio de agrupación para los compuestos activos son diferentes en cada una (tabla 2). La efectividad de la función para distinguir los compuestos activos de los inactivos en cada cepa se evaluó con el parámetro estadístico lambda de Wilks $(\lambda)$ [4].

Tabla 2. Concentración de cloroquina usada como referencia y concentración utilizada como criterio de agrupación para los compuestos activos

\begin{tabular}{ccc}
\hline Plasmodium falciparum & Concentración de referencia & Criterio de agrupamiento \\
\hline P. fal. (D6) cepa sensible & $\mathrm{EC}_{50}=0,005 \mu \mathrm{M}$ & $\mathrm{EC}_{50}<0,05 \mu \mathrm{M}$ \\
$P$. fal. (W2) cepa resistente & $\mathrm{EC}_{50}=0,18 \mu \mathrm{M}$ & $\mathrm{EC}_{50}<0,18 \mu \mathrm{M}$ \\
$P$. fal (C235) cepa resistente & $\mathrm{EC}_{50}=0,075 \mu \mathrm{M}$ & $\mathrm{EC}_{50}<0,100 \mu \mathrm{M}$ \\
\hline
\end{tabular}

La validación de estas funciones FD se realizó mediante un test interno del tipo leave some out. Para ello, el grupo de 54 compuestos en cada cepa, se dividió en 4 subgrupos: A, B, C y D, cada uno de ellos con aproximadamente el $25 \%$ de los compuestos activos e inactivos. A continuación, se realizó el ALD con los mismos IT de la FD y usando como grupo de entrenamiento tres de los cuatro subgrupos; el cuarto subgrupo se utilizó como test de predicción. Este proceso se repitió cuatro veces, intercambiando los subgrupos de entrenamiento y de predicción.

Con las FD obtenidas, se realizaron tres diagramas de distribución de la actividad antimalárica. Donde E representa la expectancia (valores en los cuales la probabilidad de encontrar compuestos activos es mayor), ubicada en el eje de las ordenadas y los valores de la FD, en el eje de las abscisas. Esta representación gráfica permite visualizar los intervalos de actividad antimalárica predicha para 
los 52 compuestos en las tres cepas, identificando las áreas de solapamiento entre los valores de actividad e inactividad y los intervalos en los valores de las FD, donde la expectancia (E) de encontrar compuestos activos es mayor [18].

De este modo, se pueden elaborar funciones discriminantes con capacidad para identificar nuevos compuestos que sean activos frente a la cepa sensible y también a las cepas resistentes.

\section{RESULTADOS Y DISCUSIÓN}

Se realizaron tres análisis discriminantes, uno para cada cepa de $P$. falciparum. Se utilizó, como criterio de agrupamiento el $\mathrm{EC}_{50}$ del fármaco de referencia, cloroquina, para cada cepa. Como variable dependiente se utilizó la variable de agrupación (A, activo e I, inactivo) y como variables independientes, los diferentes índices topológicos.

De este modo, la FD clasifica cada compuesto como activo o inactivo según el valor obtenido en la función. Un valor inferior a $0(\mathrm{FD}<0)$ hará que se clasifique como inactivo y un valor superior $(\mathrm{FD}>0)$, que se clasifique como activo, tal y como se muestra en la tabla 3.

Hay que mencionar que todas estas variables son estadísticamente significativas $(p<0,05)$ en cada análisis ALD en particular.

Tabla 3. Tabla de resultados de la clasificación por el análisis ALD comparado con el resultado experimental

\begin{tabular}{|c|c|c|c|c|c|c|c|c|c|c|}
\hline \multirow[b]{2}{*}{ Comp. ID } & \multicolumn{3}{|c|}{ P. fal. (D6) } & \multicolumn{3}{|c|}{ P. fal. (W2) } & \multicolumn{3}{|c|}{ P. fal. (C235) } & \multirow[b]{2}{*}{ Clas(global) } \\
\hline & $\mathrm{EC}^{*}{ }_{50}$ & $\mathrm{FD}_{1}$ & Clas. $^{a}$ & $\mathrm{EC}^{*}{ }_{50}$ & $\mathrm{FD}_{2}$ & Clas. $^{b}$ & $\mathrm{EC}^{*}{ }_{50}$ & $\mathrm{FD}_{3}$ & Clas. $^{c}$ & \\
\hline NEU-961 & 0,02 & 5,75 & $\mathrm{~A}$ & 0,04 & 9,47 & $\mathrm{~A}$ & 0,04 & 5,21 & A & A \\
\hline NEU-1029 & 0,01 & 0,82 & A & 0,09 & 3,55 & A & 0,03 & 3,73 & A & A \\
\hline NEU-1030 & 0,2 & $-0,01$ & I & 0,47 & $-5,30$ & I & 0,18 & $-1,73$ & I & I \\
\hline NEU-1926 & 0,02 & 2,58 & A & 0,04 & 3,69 & A & 0,03 & 4,43 & A & A \\
\hline NEU-1927 & 0,09 & $-3,32$ & I & 0,08 & 6,52 & A & 0,1 & 4,18 & A & I \\
\hline NEU-1031 & 0,06 & 0,74 & $A^{\prime}$ & 0,22 & $-5,21$ & I & 0,11 & $-1,33$ & I & I \\
\hline NEU-1019 & 0,13 & $-1,82$ & I & 0,24 & $-4,62$ & I & 0,1 & $-2,35$ & I' & I \\
\hline NEU-1020 & 2,1 & $-3,89$ & I & 4,7 & $-3,94$ & I & 4,8 & $-3,23$ & I & I \\
\hline NEU-1027 & 0,2 & $-4,83$ & I & 0,65 & $-4,02$ & I & 0,34 & $-3,60$ & I & I \\
\hline NEU-1929 & 0,02 & $-0,61$ & I' & 0,05 & $-4,67$ & I' & 0,03 & $-1,84$ & I' & I \\
\hline NEU-1930 & 0,16 & $-4,01$ & I & 0,11 & 1,60 & A & 0,21 & $-2,29$ & I & I \\
\hline NEU-1021 & 0,05 & $-2,52$ & I' & 0,12 & 4,88 & A & 0,05 & 2,22 & A & I \\
\hline NEU-1022 & 0,13 & $-2,81$ & I & 0,2 & 4,88 & $A^{\prime}$ & 0,11 & 2,04 & $A^{\prime}$ & I \\
\hline NEU-1024 & 0,12 & $-7,10$ & I & 0,66 & $-3,09$ & I & 0,22 & $-3,15$ & I & I \\
\hline NEU-1026 & 0,25 & $-1,61$ & I & 0,45 & $-4,71$ & I & 0,32 & $-2,76$ & I & I \\
\hline NEU-1025 & 0,06 & $-4,49$ & I & 0,64 & $-4,11$ & I & 0,32 & $-3,96$ & I & I \\
\hline NEU-1018 & 0,03 & 2,21 & A & 0,07 & 2,41 & A & 0,03 & 3,66 & A & A \\
\hline NEU-1028 & 0,16 & $-4,69$ & I & 0,51 & $-3,13$ & I & 0,21 & $-3,72$ & I & I \\
\hline NEU-1023 & 0,11 & $-1,22$ & I & 0,38 & $-1,69$ & I & 0,16 & $-2,04$ & I & I \\
\hline NEU-1931 & 0,01 & 4,73 & A & 0,02 & 8,96 & A & 0,02 & 4,41 & A & A \\
\hline
\end{tabular}




\begin{tabular}{|c|c|c|c|c|c|c|c|c|c|c|}
\hline \multirow[b]{2}{*}{ Comp. ID } & \multicolumn{3}{|c|}{ P. fal. (D6) } & \multicolumn{3}{|c|}{ P. fal. (W2) } & \multicolumn{3}{|c|}{ P. fal. (C235) } & \multirow[b]{2}{*}{ Clas(global) } \\
\hline & $\mathrm{EC}^{*}{ }_{50}$ & $\mathrm{FD}_{1}$ & Clas. ${ }^{a}$ & $\mathrm{EC}^{*}{ }_{50}$ & $\mathrm{FD}_{2}$ & Clas. $^{\mathrm{b}}$ & $\mathrm{EC}^{*}{ }_{50}$ & $\mathrm{FD}_{3}$ & Clas. $^{c}$ & \\
\hline NEU-1060 & 0,03 & 6,78 & A & 0,03 & 4,20 & A & 0,03 & 2,15 & A & A \\
\hline NEU-1899 & 0,1 & $-3,78$ & I & 0,19 & 3,43 & $A^{\prime}$ & 0,08 & 4,88 & A & I \\
\hline NEU-1900 & 0,02 & 0,35 & A & 0,05 & 3,05 & A & 0,02 & 4,93 & A & A \\
\hline NEU-2076 & 0,03 & 6,45 & A & 0,04 & 3,12 & A & 0,03 & 3,25 & A & A \\
\hline NEU-2152 & 0,03 & 4,61 & A & 0,03 & 4,31 & A & 0,04 & 2,04 & A & A \\
\hline NEU-2086 & 0,03 & 0,85 & A & 0,03 & 4,40 & A & 0,03 & 1,77 & A & A \\
\hline NEU-2077 & 0,24 & 0,85 & $A^{\prime}$ & 0,22 & 4,40 & $A^{\prime}$ & 0,33 & 1,79 & $A^{\prime}$ & A \\
\hline NEU-1898 & 0,05 & 2,29 & A & 0,17 & 3,99 & A & 0,07 & 4,01 & A & A \\
\hline NEU-1953 & 0,03 & 0,49 & A & 0,06 & $-0,50$ & I' & 0,04 & 0,77 & A & I \\
\hline NEU-1956 & 0,04 & 1,28 & A & 0,07 & 1,97 & A & 0,04 & 1,09 & A & A \\
\hline NEU-1957 & 0,09 & $-1,29$ & I & 0,18 & 5,93 & A & 0,1 & 5,66 & A & I \\
\hline NEU-2088 & 0,94 & $-5,27$ & I & 2,1 & $-5,34$ & I & 1,4 & $-6,11$ & I & I \\
\hline NEU-2087 & 0,25 & $-8,10$ & I & 0,22 & $-6,75$ & I & 0,53 & $-5,30$ & I & I \\
\hline NEU-2096 & 0,13 & $-2,60$ & I & 0,4 & $-1,76$ & I & 0,21 & 2,28 & $A^{\prime}$ & I \\
\hline NEU-2091 & 0,05 & $-5,85$ & I' & 0,1 & $-0,49$ & I' & 0,09 & 0,56 & A & I \\
\hline NEU-2090 & 0,1 & $-3,58$ & I & 0,24 & 2,18 & $A^{\prime}$ & 0,16 & 1,23 & $A^{\prime}$ & I \\
\hline NEU-2094 & 0,18 & $-5,60$ & I & 0,52 & $-0,49$ & I & 0,37 & 0,61 & $A^{\prime}$ & I \\
\hline NEU-2093 & 0,08 & $-3,98$ & I & 0,16 & 2,18 & A & 0,08 & 1,11 & A & I \\
\hline NEU-2129 & 0,5 & $-4,69$ & I & 1,5 & $-5,54$ & I & 0,76 & $-5,78$ & I & I \\
\hline NEU-2130 & 0,16 & $-7,39$ & I & 0,63 & $-6,91$ & I & 0,2 & $-4,63$ & I & I \\
\hline NEU-2131 & 0,3 & $-4,90$ & I & 0,6 & $-1,60$ & I & 1,4 & $-6,85$ & I & I \\
\hline NEU-2133 & 1 & $-4,46$ & I & 2,6 & $-5,50$ & I & 1,2 & $-6,63$ & I & I \\
\hline NEU-2134 & 0,1 & $-5,89$ & I & 0,44 & $-3,82$ & I & 0,17 & $-3,80$ & I & I \\
\hline NEU-2142 & 1,2 & $-7,29$ & I & 6,9 & $-6,01$ & I & 5,9 & $-3,98$ & I & I \\
\hline NEU-2143 & 0,4 & $-1,29$ & I & 2,3 & $-5,80$ & I & 1,9 & $-4,56$ & I & I \\
\hline NEU-2144 & 0,6 & $-4,90$ & I & 5,9 & $-5,72$ & I & 4 & $-5,01$ & I & I \\
\hline NEU-2147 & 0,86 & $-4,87$ & I & 5,5 & $-5,80$ & I & 5,5 & $-5,25$ & I & I \\
\hline NEU-2146 & 6,8 & $-7,52$ & I & 21 & $-6,05$ & I & 13 & $-3,90$ & I & I \\
\hline NEU-2148 & 0,03 & $-1,31$ & I' & 0,28 & $-7,08$ & I & 0,14 & $-5,26$ & I & I \\
\hline NEU-2141 & 0,63 & $-2,52$ & I & 1,7 & $-6,36$ & I & 1 & $-7,31$ & I & I \\
\hline NEU-2149 & 0,05 & $-1,31$ & I' & 0,21 & $-6,98$ & I & 0,12 & $-7,06$ & I & I \\
\hline NEU-2150 & 0,09 & $-1,40$ & I & 0,56 & $-5,04$ & I & 0,25 & $-4,68$ & I & I \\
\hline NEU-2145 & 0,18 & $-6,36$ & I & 0,95 & $-4,95$ & I & 0,37 & $-4,98$ & I & I \\
\hline NEU-2151 & 0,11 & $-5,12$ & I & 0,34 & $-5,21$ & I & 0,22 & $-4,07$ & I & I \\
\hline Cloroquina & 0,01 & & & 0,18 & & & 0,08 & & & \\
\hline
\end{tabular}

*Valores experimentales extraídos del artículo de Mehta et. al [10]

${ }^{a}$ Clasificación obtenida a partir del valor de $\mathrm{FD}_{1}$, Ec. 1.

${ }^{\mathrm{b}}$ Clasificación obtenida a partir del valor de $\mathrm{FD}_{2}$, Ec. 2.

${ }^{\mathrm{c}}$ Clasificación obtenida a partir del valor de $\mathrm{FD}_{3}$, Ec. 3 .

${ }^{\mathrm{d}}$ Clasificación global obtenida a partir de los valores de $\mathrm{FD}_{1}, \mathrm{FD}_{2}$ y $\mathrm{FD}_{3}$.

' Compuestos clasificados erróneamente por las funciones discriminantes $\mathrm{FD}_{1}, \mathrm{FD}_{2}$ y FD $\mathrm{FD}_{3}$. 
Para el primer grupo (P. falciparum $\mathrm{D} 6$, cepa sensible a la cloroquina) se obtuvo la ecuación $\mathrm{FD}_{1}$ :

$$
\begin{aligned}
& \mathrm{FD}_{1}=-199,4+40,2 \text { BEHv3 }-13,9 \mathrm{GGI} 7+19,9 \mathrm{GGI} 5-28,3 \text { BELe } 2+424,2 \mathrm{MSD}-6,2 \text { EEig02d } \\
& \mathrm{N}=54 \quad \lambda(\text { Lambda de Wilks) }=0,432 \quad \mathrm{~F}(6,47)=10,3 \quad \mathrm{p}<0,00001
\end{aligned}
$$

En la ecuación Ec.1 los índices topológicos que aparecen son: BEHv3, que indica el valor propio más bajo obtenido con la matriz de Burden ponderada por los volúmenes atómicos de Vander Waals (valores propios de Burden); GGI7 y GGI5, que representan los índices topológicos de carga de orden siete y cinco, respectivamente; BELe2, que corresponde con el valor propio más bajo obtenido con la matriz de Burden ponderada por las electronegatividades atómicas de Sanderson (valores propios de Burden); MSD, el índice de distancia cuadrático medio de Balaban (descriptor topológico) y EEig02d, que es el valor propio de orden dos obtenido a partir de la matriz de adyacencia de aristas ponderada por los momentos dipolares (índices de adyacencia de aristas).

De los 54 compuestos utilizados en el análisis, 15 se clasificaron como activos, de los cuales 2 estaban mal clasificados, ya que experimentalmente eran inactivos y 39 inactivos, de los cuales 5 estaban mal clasificados, puesto que se demostraron activos experimentalmente.

Por último, a partir de la matriz de clasificación, se calcularon la sensibilidad $(81,25 \%)$, la especificidad $(94,74 \%)$ y la exactitud $(90,74 \%)$ de esta $\mathrm{FD}_{1}$. Estos datos corroboran la capacidad discriminante de la función $\mathrm{FD}_{1}$.

Para el segundo grupo (P. falciparum W2, cepa resistente a la cloroquina), se obtuvo la ecuación $\mathrm{FD}_{2}$ :

$$
\begin{array}{ll}
\mathrm{FD}_{2}=-229,9+0,043 \mathrm{ZM} 2 \mathrm{~V}+0,257 & \mathrm{~T}(\mathrm{O} . . \mathrm{O})+3692,2 \mathrm{VRv} 2 \\
\mathrm{~N}=54 & \lambda(\text { Lambda de Wilks })=0,340 \quad \mathrm{~F}(4,49)=23,8 \quad \mathrm{p}<0,7 \mathrm{VRD} 2 \\
&
\end{array}
$$

Los índices topológicos presentes en la ecuación son: ZM2V, índice de Zagreb de valencia de orden dos (un descriptor topológico); $\mathrm{T}(\mathrm{O} . . \mathrm{O})$, suma de las distancias topológicas entre oxígenos (descriptor topológico) y VRv2, VRD2 que son índices basados en los valores propios de la matriz de distancia.

Se clasificaron 54 compuestos, de los cuales 21 se englobaron como compuestos activos, pero 2 estaban mal clasificados (experimentalmente eran inactivos); asimismo, 33 se clasificaron como inactivos, 3 de ellos estaban mal clasificados, pues eran activos experimentalmente.

Para terminar, a partir de la matriz de clasificación elaborada con los datos anteriores, se calculó la sensibilidad (86,36 \%), especificidad (93,75\%) y exactitud (90,74 \%). Observando los resultados, podemos ratificar la capacidad discriminante de la función correspondiente a la ecuación 2.

Para el tercer grupo (P. falciparum $\mathrm{C} 235$, cepa resistente a la cloroquina), se obtuvo la siguiente ecuación $\mathrm{FD}_{3}$ :

$$
\begin{array}{llll}
\mathrm{FD}_{3}=20,40-26,51 \mathrm{JhetZ}+0,18 \mathrm{~T}(\mathrm{O} . . \mathrm{O}) & -0,021 \mathrm{D} / \mathrm{Dr} 06+17,54 & \mathrm{BELe} 3 \\
\mathrm{~N}=54 & \lambda(\text { Lambda de Wilks })=0,398 & \mathrm{~F}(4,49)=18,5 & \mathrm{p}<0,00001
\end{array}
$$

En esta ecuación las variables son cuatro descriptores topológicos: JhetZ o índice tipo Balaban, $\mathrm{T}(\mathrm{O} . . \mathrm{O})$, que indica la suma de las distancias topológicas entre oxígenos, y D/Dr06, correspondiente al índice de distancia de ciclo de orden seis; además, de un valor propio de Burden que es el BELe3, valor propio más bajo obtenido con la matriz de Burden ponderada por las electronegatividades atómicas de Sanderson. 
Se utilizaron 54 compuestos para la clasificación y 24 se catalogaron como activos. Sin embargo, 5 estaban mal catalogados puesto que experimentalmente eran inactivos y los 30 restantes se clasificaron como inactivos, aunque 2 estaban erróneamente clasificados porque experimentalmente se demostraron activos.

Después se determinó la sensibilidad (90,48\%), la especificidad (84,85\%) y la exactitud $(87,037 \%)$, calculadas a partir de la matriz de clasificación anteriormente descrita. Al igual que en las ecuaciones uno y dos, estos resultados confirman la capacidad discriminante de la Ec. 3.

Todas las ecuaciones FD se validaron mediante un test interno del tipo leave some out. Los resultados obtenidos en el test de discriminación fueron similares a la FD obtenida y seleccionada para cada cepa.

Una vez obtenidos los resultados para las tres cepas se realizó un diagrama de distribución de la actividad antimalárica para cada una de ellas por separado (figura 1), en donde se pueden observar bandas blancas correspondientes al grupo inactivo y bandas negras que se corresponden al grupo activo. En estos diagramas, se puede distinguir cierto solapamiento entre ambos grupos, por ello la mayor probabilidad de encontrar compuestos activos la situaremos entre el 0 y el 8 para la $\mathrm{FD}_{1}$, para $1 \mathrm{~F} \mathrm{FD}_{2}$ entre el 0 y el 10 y finalmente para la $\mathrm{FD}_{3}$ entre el 0 y el 6 . En estos rangos o dominios de aplicación, estarán contenidos los compuestos antimaláricos seleccionados por el modelo, frente a las cepas de P. falciparum resistentes (W2 y C325) y frente a la cepa sensible (D6) simultáneamente con la cloroquina.
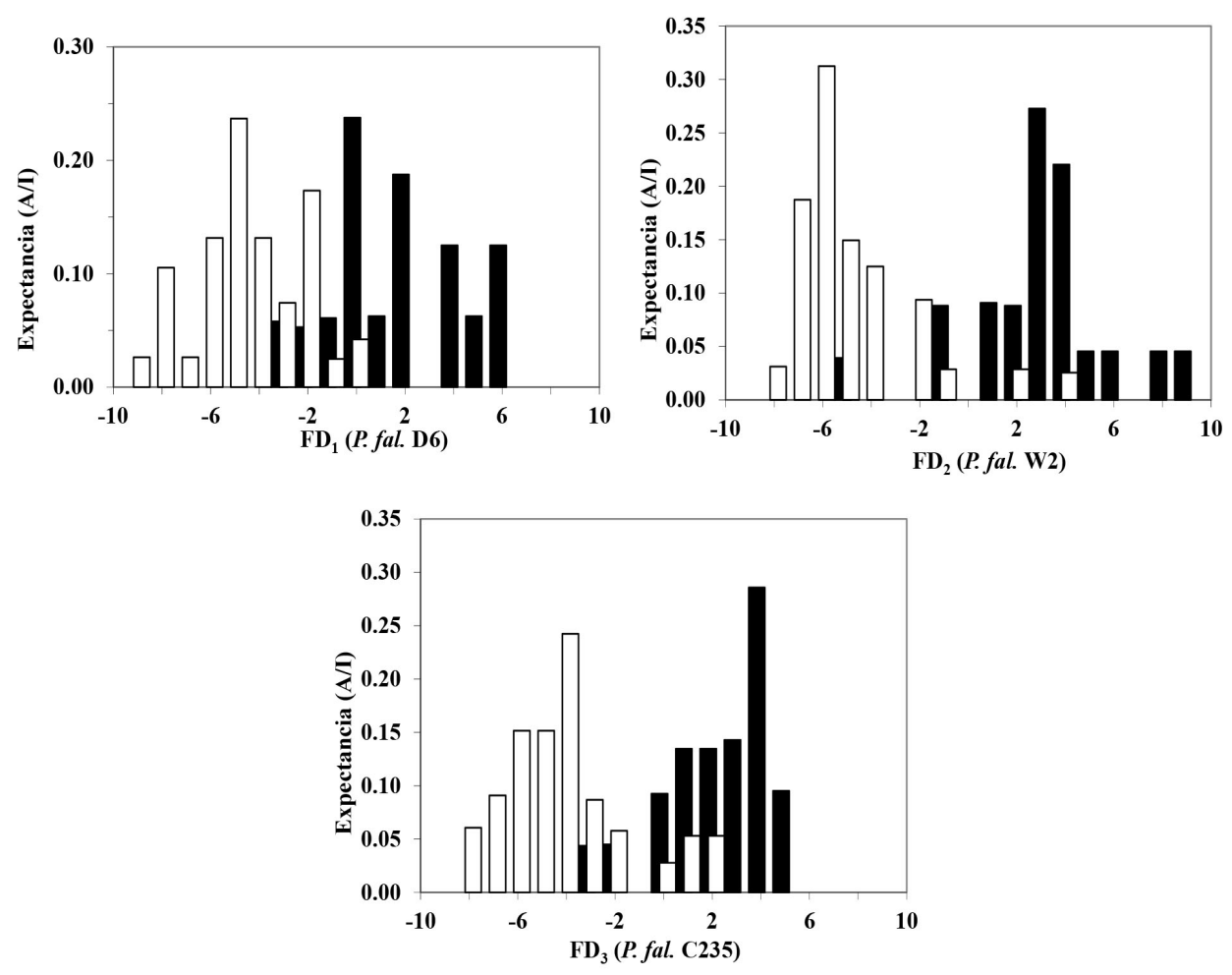

Fig. 1. Diagramas de distribución de la actividad antimalárica frente a $P$. fal. D6, P. fal. W2 y P. fal. $\mathrm{C} 235$, obtenidos con las funciones $\mathrm{FD}_{1}, \mathrm{FD}_{2}$ y $\mathrm{FD}_{3}$ (blanco, grupo inactivo y negro, grupo activo) 
Por tanto, el modelo topológico propuesto frente a $P$. falciparum deberá seleccionar compuestos con valores de actividad de $\mathrm{EC}_{50}$ frente a las cepas D6, W2 y $\mathrm{C} 235$ inferiores a $0,05,0,18$ y $0,1 \mu \mathrm{M}$, respectivamente.

Este modelo, se utilizó para realizar un screening virtual, con el fin de proponer nuevos compuestos potencialmente activos. Para ello, se utilizó la base PubChem, la cual contiene más de 100 millones de moléculas o compuestos.

Se partió de los compuestos más activos como el Neu-961 y otros seleccionados por el modelo topológico discriminante y se buscaron compuestos análogos a ellos.

Se trabajó con alrededor de 2.500 compuestos, a los cuales se les calcularon los diferentes índices topológicos con el programa Dragon a partir del código Smiles. Posteriormente, se les pasó el modelo topológico seleccionado. De los 2.500 compuestos, solo el $2 \%$ superaron los tres filtros (las tres ecuaciones FD), como son los compuestos de la tabla 4, en la que se muestran algunas estructuras.

Tabla 4. Estructura química de algunos de los compuestos seleccionados por el modelo topológico desarrollado

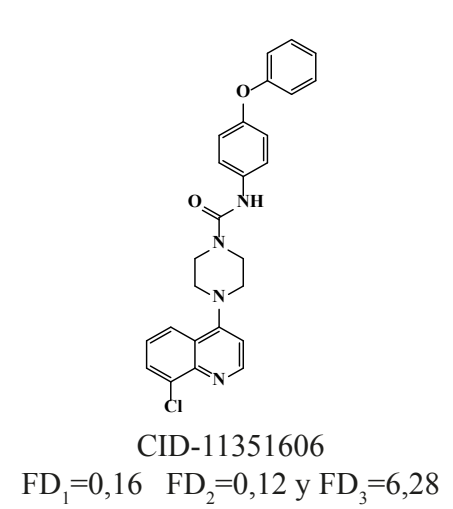

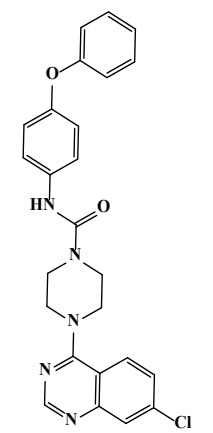

CID-22707359

$\mathrm{FD}_{1}=0,06 \quad \mathrm{FD}_{2}=0,68$ y $\mathrm{FD}_{3}=6,28$
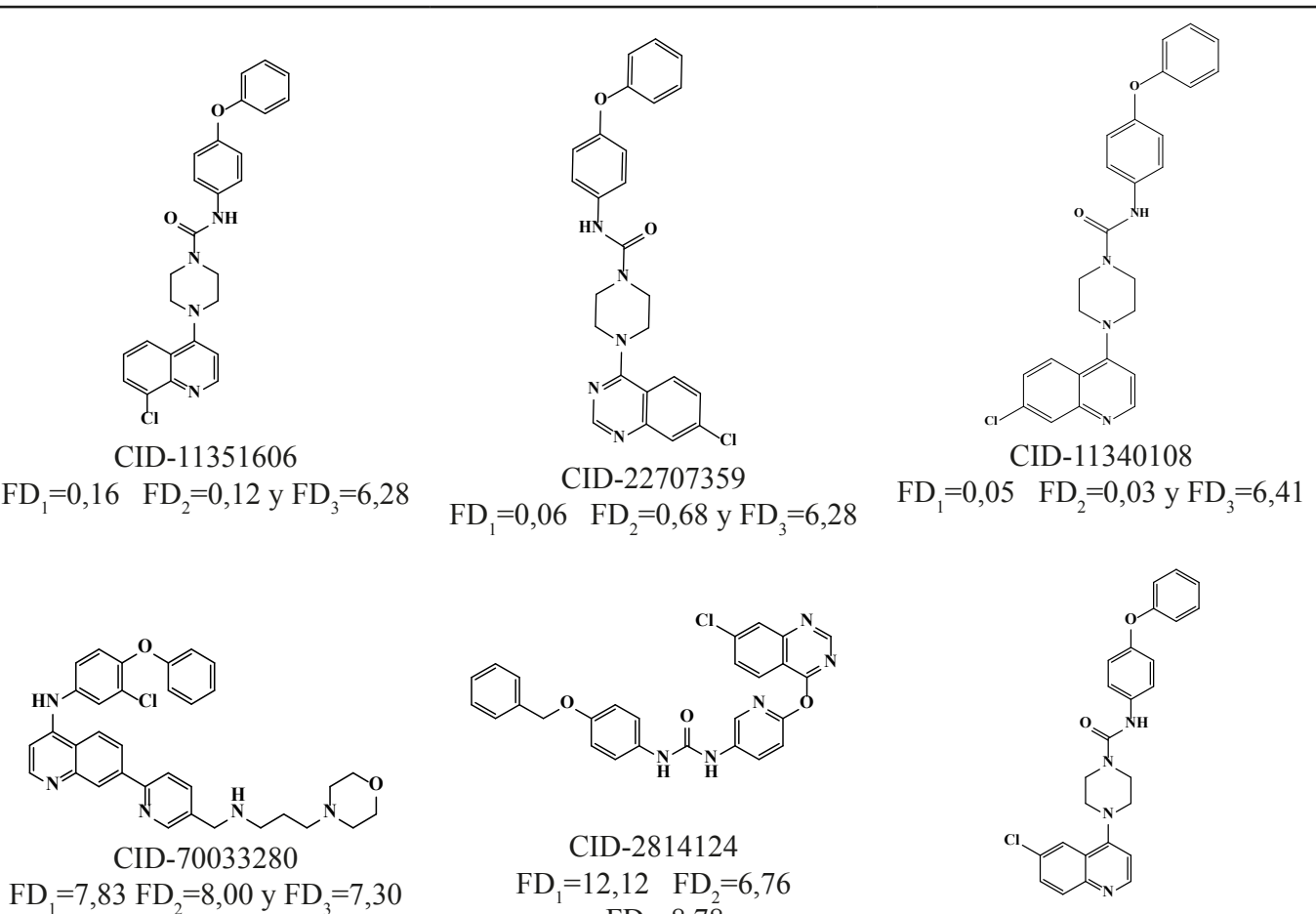

CID-2814124

$\mathrm{FD}_{1}=12,12 \quad \mathrm{FD}_{2}=6,76$
$\mathrm{y} \mathrm{FD}_{3}=8,78$

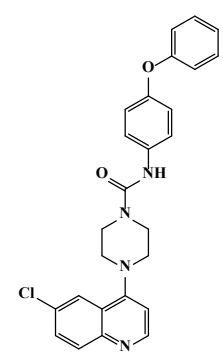

CID-11419763

$\mathrm{FD}_{1}=0,91 \quad \mathrm{FD}_{2}=0,08$ y $\mathrm{FD}_{3}=6,26$

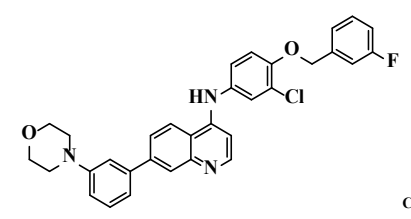

CID-118364939

$\mathrm{FD}_{1}=3,08 \quad \mathrm{FD}_{2}=7,90$ y $\mathrm{FD}_{3}=6,05$

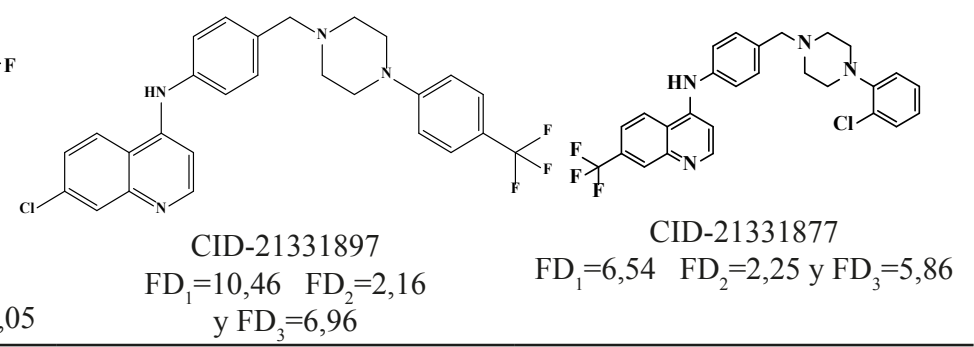


Para corroborar las actividades de los compuestos de la tabla 4 y otros que demuestran tener actividad según el análisis ALD, se debería posteriormente obtener los compuestos en un laboratorio y realizar ensayos in vitro que demuestren la actividad predicha mediante la topología molecular.

La topología es una herramienta más en el arduo trabajo de encontrar nuevos fármacos activos frente a enfermedades importantes como es el caso de la malaria.

Cabe destacar que los compuestos seleccionados mediante el modelo topológico ya han sido probados en bioensayos, donde demuestran su utilidad en diferentes campos de la medicina. Como es el caso de los compuestos CID-11351606, CID-22707359, CID-11340108, CID-11419763, que han demostrado ser inhibidores de la fosforilación del receptor de PDGF [19], lo que ayuda a combatir el crecimiento anormal en las células, siendo de gran utilidad para la prevención o el tratamiento de enfermedades celulares proliferativas, de manera que estos compuestos pueden tener una función antineoplásica, entre otras [20]. El compuesto CID-2814124 también se considera de gran utilidad para el desarrollo de medicamentos contra el cáncer gracias a su actividad como inhibidor de determinadas acciones enzimáticas [21]. Otros, además, forman parte de compuestos que son útiles en el tratamiento de la osteoporosis y la poliquistosis renal, como es el caso del CID-70033280 [22] o antihipertensivos en el caso de CID-21331897 y CID-21331877 [23].

Por último, cabe resaltar el compuesto CID-11836939, del que, en un bioensayo, evaluaron su actividad como inhibidor del crecimiento parasitario durante 72 horas; el ensayo se realizó mediante fluorescencia verde SYBR y los resultados indican que este compuesto presenta actividad antimalárica contra Plasmodium falciparum (D6, C235, W2) [24]. También resultó ser activo frente a otros parásitos, tales como: formas amastigotes de Leishmania major o Trypanosoma brucei [24]. Los resultados experimentales con este compuesto realzan el valor del presente trabajo, puesto que demuestran que un compuesto considerado activo por el modelo topológico también ha sido activo en otros ensayos experimentales, lo que confirma la validez de la predicción.

Sin embargo, se necesitan más ensayos complementarios a este análisis teórico, en los cuales se lleve a la práctica y se demuestren estos resultados, para así dar más valor a estos.

\section{CONCLUSIONES}

Los resultados obtenidos del análisis de 54 compuestos análogos de la 4-anililquinolina muestran que el análisis QSAR basado en la topología molecular es una metodología muy útil y eficaz en la búsqueda de nuevos compuestos antimaláricos. Se aplicó un modelo matemático basado en el análisis lineal discriminante para cada una de las tres cepas de Plasmodium falciparum (una sensible a la cloroquina y dos resistentes) que determinó si los compuestos tenían actividad antimalárica o no en cada caso. Con este modelo de tres funciones (Ec. 1, Ec. 2 y Ec. 3) se realizó un screening virtual en el que se propusieron nuevos compuestos potencialmente activos frente a las tres cepas. Esto hace esperar que los modelos descritos en este trabajo puedan utilizarse para el rastreo en bases moleculares que permitan presentar nuevos fármacos útiles para la lucha contra la malaria. 


\section{AGRADECIMIENTOS}

A. E. P., R. P., J. M. y A. U. agradecen al máster en Enfermedades Parasitarias Tropicales de la Universitat de València por haber permitido realizar este trabajo. J. G. y R. G. D agradecen al proyecto PID2019-107464RB-C22, "Diseño racional de nuevas herramientas de fitoprotección (Newcropproctools)" concedido por el Ministerio de Ciencia e Innovación de España por el material utilizado para la realización de este trabajo.

\section{REFERENCIAS BIBLIOGRÁFICAS}

[1] World Health Organization. World Malaria Report: 20 years of global progress and challenges. Vol. WHO/HTM/GM, World Health. 2020. 238 p.

[2] Paludismo. [consultado: 27 de enero 2021]. Disponible en: https://www.who.int/es/news-room/ fact-sheets/detail/malaria

[3] World Health Organization. Malaria 2016-2030. 2016;35. Disponible en: http://apps.who.int/ iris/bitstream/10665/186671/1/9789243564999_spa.pdf

[4] Gálvez J, Gálvez-Llompart M, García-Doménech R. Molecular topology as a novel approach for drug discovery. Expert Opinion on Drug Discovery. 2012;7(2):133-153. DOI:10.1517/1746 0441.2012.652083.

[5] Mahmoudi N, García-Doménech R, Gálvez J, Farhati K, Franetich JF, Sauerwein R, et al. New active drugs against liver stages of Plasmodium predicted by molecular topology. Antimicrob Agents Chemother. 2008 Apr;52(4):1215-1220.

[6] Baptista-Peraza I, Otero-Pérez C, González-Apráez S, Pertegás-Sevilla A, Gálvez J, GarcíaDoménech R. Aplicación de la Topología Molecular al Análisis de la Actividad Antimalárica de 4-Aminobiciclo [2.2. 2] Octan-2-il 4-Aminobutanoatos y sus Análogos Etanoatos y Propanoatos. Nereis. Interdisciplinary Ibero-American Journal of Methods, Modelling and Simullation. 2019; 11: 51-64.

[7] Carrillo JS, Rizza C, Álvarez BE, Hernández D, Gálvez J, García-Doménech R. Aplicación de la Topología Molecular en la búsqueda de nuevos compuestos basados en Azaauronas derivados de las Auronas naturales como potenciales antimaláricos. Nereis. Interdisciplinary Ibero-American Journal of Methods, Modelling and Simulation. 2017; 9:49-62.

[8] García-Doménech R, Zanni R, Gálvez-Llompart M, Gálvez J. Computational design of chemical for the control of mosquitoes and their diseases. CRC Press Taylor \& Francis Group. 2018; 4:107-137.

[9] Gálvez J, de Julián-Ortiz J, García-Doménech R. Application of molecular topology to the prediction of potency and selection of novel insecticides active against malaria vectors, J. Molec. Struct. Theochem. 2005;727:107-113 (esp. 11-13).

[10] Mehta N, Ferrins L, Leed SE, Sciotti RJ, Pollastri MP. Optimization of Physicochemical Properties for 4-Anilinoquinoline Inhibitors of Plasmodium falciparum Proliferation. ACS Infect Dis. 2018;4(4):577-91. DOI: 10.1021/acsinfecdis.7b00212

[11] Dassault Systèmes. BIOVIA Draw (R) Versión MDL.Draw.Editor 17.1.0.900. 2016. 
[12] Dragon para Windows (software para cálculos de descriptores moleculares) versión 5.0. Talete SRL. Milán, Italia, 2004.

[13] Gálvez J, García-Doménech R, Salabert MT, Soler R. Charge indexes. New topological Descriptors. J Chem Inf Comput Sci. 1994;34:520-525.

[14] Balaban AT. Highly discriminating distance-based topological index. Chemical Physics Letters 1982;89(5):399-404.

[15] Zanni R, Gálvez-Llompart M, García-Doménech R, Galvez J. Latest advances in molecular topology applications for drug discovery. Expert Opin Drug Discov. 2015;10(9):945-57.

[16] García-Doménech R, López-Peña W, Sanchez-Perdomo Y, Sanders JR, Sierra-Araujo MM, Zapata $\mathrm{C}$, et al. Application of molecular topology to the prediction of the antimalarial activity of a group of uracil-based acyclic and deoxyuridine compounds. Int J Pharm 2008;363(1):78-84.

[17] Statistica. StatSoft Inc. STATISTICA (Data Analysis Software System). 2007.

[18] Gálvez J, García-Doménech R, De Gregorio Alapont C, De Julián-Ortiz JV, Popa L. Pharmacological distribution diagrams: A tool for de novo drug design. J Mol Graph 1996;14(5):272-276. DOI: 10.1016/s0263-7855(96)00081-1

[19] Matsuno K, Ushiki J, Seishi T, Ichimura M, Giese NA, Yu J, et al. Potent and selective inhibitors of platelet-derived growth factor receptor phosphorylation. 3. Replacement of quinazoline moiety and improvement of metabolic polymorphism of 4-[4-(N-substituted (thio)carbamoyl)1-piperazinyl]-6,7-dimethoxyquinazoline derivatives. J Med Chem. 2003-11-06;46(23):49104925.

[20] Matsuno K, Ichimura M, Nomoto Y, Sujiwara S, Ide S, Tsukuda E, Irie J, Oda S, inventores; Kyowa Kirin Co Ltd, cesionario. Nitrogen-containing heterocyclic compounds. Canadian patent CA 2239227. 1998 Apr 9.

[21] Kumar A, Ito A, Hirohama M, Yoshida M, Zhang KYJ. Identification of quinazolinyloxy biaryl urea as a new class of SUMO activating enzyme 1 inhibitors. Bioorg Med Chem Lett. 2013-0915;23(18):5145-5149.

[22] Boschelli D, Yanong W, Boschelli F, Baerger D, Zhang N, Powell D, Ye F, Yamashita A, DeMorin F, Wu B, Tsou H, Overbeek-Klumpers E, Wissner A, inventores; Wyeth LLC, cesionario. 3-cyanoquinolines, 3-cyano-1,6-naphthyridines, and 3-cyano-1,7-naphthyridines as protein kinase inhibitors. United States Patent, US 6521618B2. 2001 Mar 28.

[23] McCall JM, inventor; Upjohn Co, cesionario. Piperazino methyl phenyl aminoquinolines. United States Patent, US 4140775A. 1979 Feb 20

[24] Devine W, Woodring JL, Swaminathan U, Amata E, Patel G, Erath J, et al. Protozoan Parasite Growth Inhibitors Discovered by Cross-Screening Yield Potent Scaffolds for Lead Discovery. J Med Chem. 2015-07-23;58(14):5522-5537. 